\title{
Kurtosis Based Techniques for Signal Extraction
}

\author{
Yongjian Zhao ${ }^{1, \mathrm{a}}$, Manlan $\mathrm{Hao}^{2, \mathrm{~b}}$ \\ ${ }^{1}$ School of Information Engineering, Shandong University (weihai), China \\ ${ }^{2}$ Wenshang County People's Hospital, Wenshang, China \\ ajian123cn@sdu.edu.cn, bjht79@163.com
}

Keywords: Independence, Separation, Mixture, Source, Kurtosis.

\begin{abstract}
As a practical measure of non-Gaussianity, kurtosis generally represents the preferred technique for signal extraction. Here a learning rule is presented associated with kurtosis. It can recover one source signal owning the maximum absolute value of kurtosis among all original sources. Computer simulations on biomedical signals demonstrate its efficiency.
\end{abstract}

\section{Introduction}

Blind source separation (BSS) is a popular statistical technique which aims at recovering all original sources from their mixtures simultaneously [1-3]. Due to its wide range of potential applications, BSS technique has been studied extensively in the domain of biomedical engineering, speech enhancement and telecommunications. In recent years, BSS has become one of the most important research topics in the signal processing area. The major drawback of conventional BSS technique is that we must extract all independent components from their mixtures. In many applications especially in biomedical signal processing, someone is only interested in one or a few original sources [4,5]. For example, in EEG or MEG we can obtain more than 64 sensor signals but only a few signals (e.g. the periodically evoked brain potentials) are desired while the rest are considered interfering noise. For such applications, separating all original sources can take a long time, either in minutes or hours basis. In other words, conventional BSS technique introduces large computational burden for a large number of mixtures. Therefore, it is important to introduce a reliable method to extract only the desired source signal that contains valuable information.

The central limit theorem tells that the distribution of a sum of independent random variables tends to a Gaussian distribution. In recent years, non-Gaussianity has become actually of paramount important in blind signal estimation [6]. As a practical measure of non-Gaussianity, kurtosis generally represents the preferred technique for signal extraction. Recently, this technique has received much research attention in the domain of applied mathematics, neural networks and statistical signal processing [2,7]. After calculating the performance of kurtosis, a new learning rule is presented in this paper associated with kurtosis. Someone can recover one source signal owning the maximum absolute value of kurtosis among all original sources. Therefore, much of the unnecessary computation load is avoided. This is particularly beneficial when the number of original sources is large and only one or a few is desired. It is worth mentioning that the signal extraction process can be derived without any precise knowledge of the original sources. In contrast to the simultaneous BSS for multiple sources, this work can provide more freedom in signal separation. Simulation results on biomedical signals demonstrate the performance of the derived method.

\section{Proposed Algorithm}

Denote the observed sensor signals $x(k)=\left[x_{1}(k), \cdots, x_{n}(k)\right]^{T}$ described by the following matrix equation

$$
x(k)=A s(k)
$$


where $A$ is an $\mathrm{n} \times \mathrm{n}$ unknown mixing matrix, $\mathrm{k}$ is the discrete time index, and $s(k)=\left[s_{1}(k), \cdots, s_{n}(k)\right]^{T}$ is a vector of unknown temporally correlated source signals which are mutually independent, zero-mean and unit-variant. Here only the vectors $\mathrm{x}(\mathrm{k})$ can be observed and everything else is unknown. When source signals are independent, BSS methods can be conveniently used to perform the signal separation. Conventional BSS technique aims to recover all source signals from their mixture simultaneously [2,6]. As the mixture of independent Gaussian sources mixed by an orthogonal mixing matrix remains independent, the BSS techniques are only applicable to non-Gaussian sources, or when at most only one source is Gaussian[7,8]. Under this condition, the extraction of one original source signal is equivalent to extracting an independent component from the mixtures. An efficient technique is to introduce an iterative process to find a weight vector $w$ so that $y(k)=w^{T} x(k)=g^{T} s(k)$ is a good approximation to the desired source signal [4,5]. Here $g^{T}=w^{T} A$ is the global demixing vector.

Non-Gaussianity is actually of paramount importance in BSS estimation [1,4]. Without non-Gaussianity, the BSS estimation is not possible at all [4,5]. To extract one source signal exclusively, it is sufficient to maximize/minimize the non-Gaussianity of $y(k)$ in (1). Our work starts by intuitively motivating the maximization of non-Gaussianity according to the central limit theorem. To use non-Gaussianity in BSS estimation, one must have a quantitative measure about non-Gaussianity of a random variable. As a first practical measure of non-Gaussianity, Kurtosis is the name given to the fourth-order cumulant of a random variable. The kurtosis of y, denoted by kt(y), is defined by

$$
k t(y)=E\left\{y^{4}\right\}-3\left(E\left\{y^{2}\right\}\right)^{2}
$$

where $E\{\cdot\}$ denotes the statistical expectation operator.

For a Gaussian signal y, the fourth moment is equal to $E\left\{y^{4}\right\}-3$. In other words, kurtosis is zero for a Gaussian random variable. The kurtosis of a Gaussian random variable is zero, whereas for most non-Gaussian random variables it is non-zero. Random variables with positive kurtosis are called super-Gaussian, whereas they are termed sub-Gaussian for a negative kurtosis. Typically non-Gaussianity can be measured by the absolute value of kurtosis. These measures are zero for a Gaussian variable, and greater than zero for most non-Gaussian random variables.

To maximize the absolute value of kurtosis, we start from certain vector $w$, compute the direction in which the absolute value of the kurtosis of $y=w^{T} x$ is growing most strongly, on the basis of the available sample $x(1), \cdots, x(T)$ of mixture vector $x$, and then move the vector $w$ in that direction. We formulate the above criterion as

$$
\text { maximize } J(w)=-\frac{\beta k t(y)}{4\left(E\left\{y^{2}\right\}\right)^{2}}
$$

where $\beta=1$ for the extraction of source signals with positive kurtosis and $\beta=-1$ for sources with negative kurtosis.

Applying the standard gradient descent approach to (3), we conduct the following new learning rule:

where $\mu$ is the step size and

$$
w(k+1)=w(k)+\mu \varphi(y(k)) x(k)
$$

$$
\varphi(y(k))=\beta\left[\frac{m_{2}(y)}{m_{4}(y)} y^{3}(k)-y(k)\right]
$$

with

$$
m_{q}(y)(k)=(1-\delta) m_{q}(y)(k-1)+\delta|y(k)|^{q}, \mathrm{q}=2,4
$$

where $\delta$ is the forgetting factor. 


\section{Simulation Results}

The ABio7 dataset proposed by Allan Barros, which can be found in the ICALAB toolbox[9], is a benchmark contains 7 typical biological signals[7,9]. Each signal has 5000 samples with zero means and unit variances. Due to space limitations, only four source signals, shown in Fig.1, were adopted. From top to down, they were, respectively, a colored Gaussian signal, an ECG, an electrode artifact and a respiratory artifact. Their normalized kurtosis were $0.04,37,0.01$ and -0.5 , respectively. A $4 \times 4$

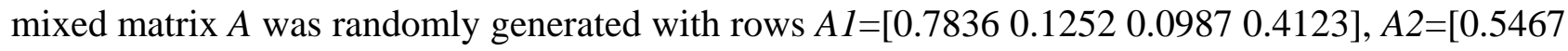
0.20130 .21530 .3765 ], $A 3=\left[\begin{array}{lllllll}0.1674 & 0.6543 & 0.5654 & 0.3432\end{array}\right]$ and $A 4=\left[\begin{array}{llllll}0.3845 & 0.1123 & 0.4987 & 0.8768\end{array}\right]$.

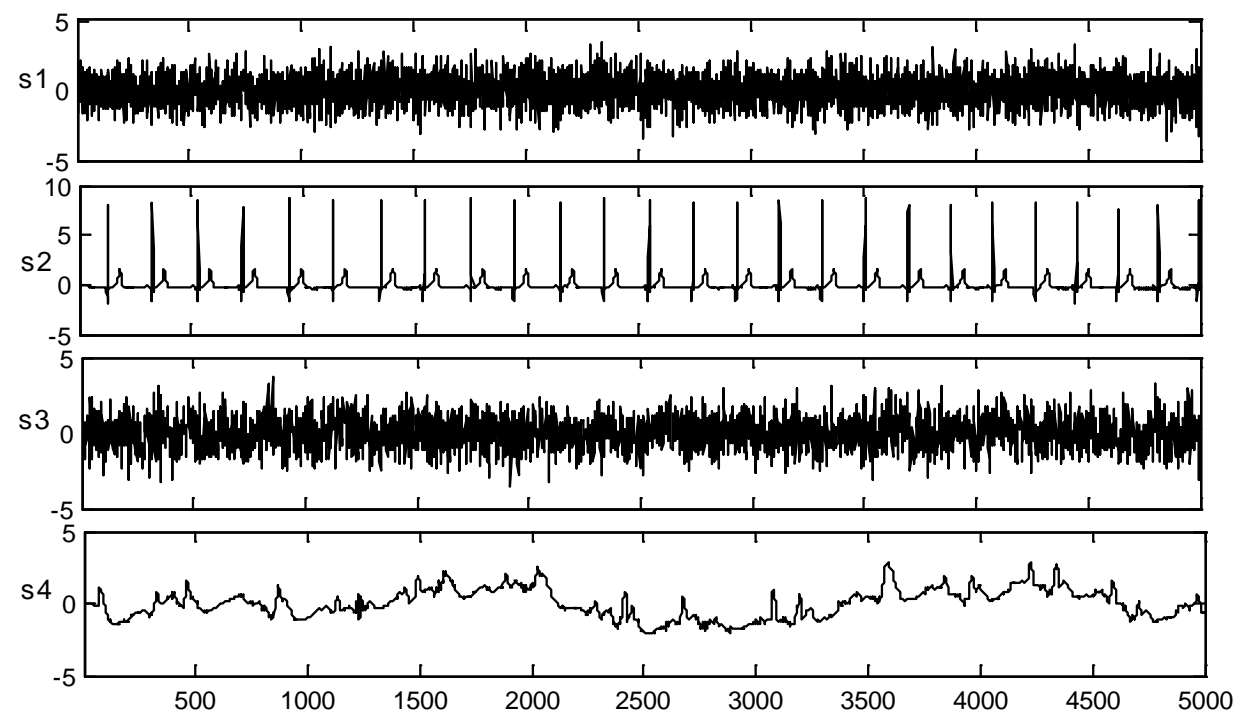

Fig.1 Four typical biomedical source signals

Source signals depicted in Fig.1 were mixed by the matrix A. The mixed results are shown in Fig.2.

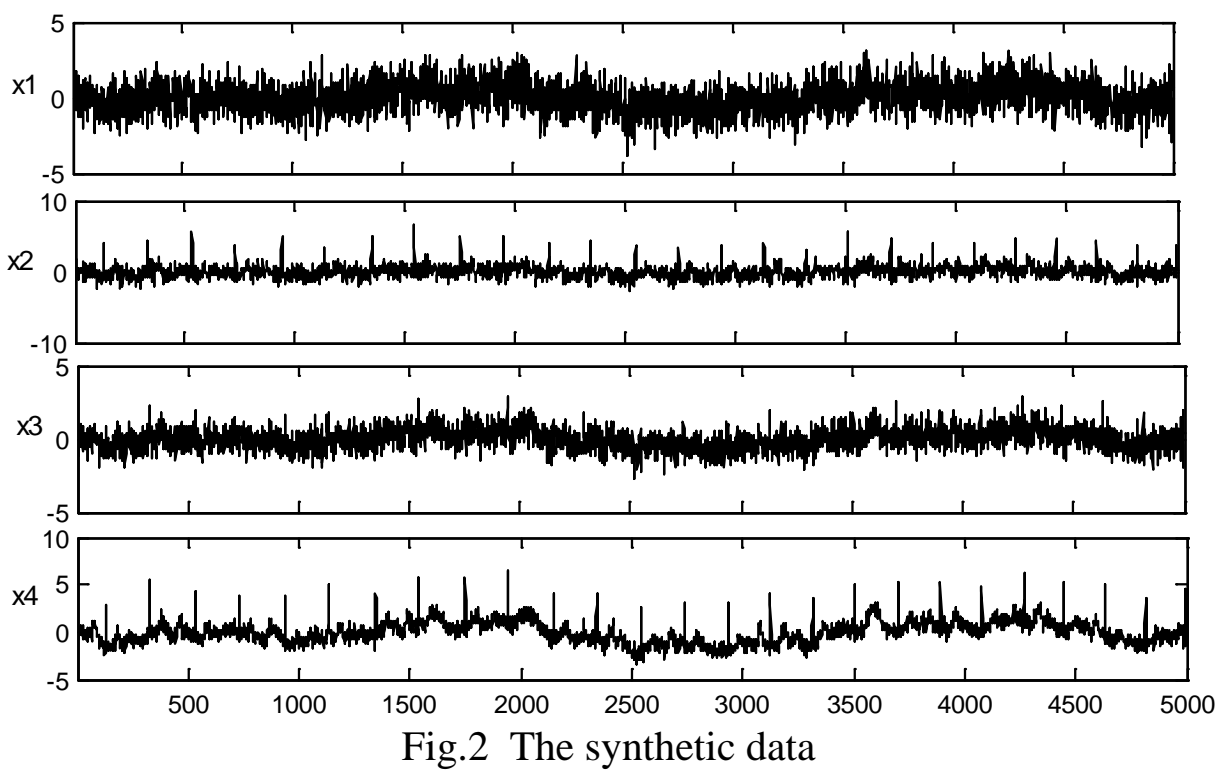

ECG signal has valuable information for clinical medicine. In practical applications, ECG signal is often contaminated by other signals or noise. Here we aim to extract the desired ECG from the synthetic data exclusively. To make comparison, we ran the following algorithms: BCBSE [2], EVBSE [4] and the proposed algorithm in this paper. The extraction results are shown in Fig.3, which are in accordance with the sequence of these algorithms. Obviously, the BCBSE algorithm shows the 
poorest performance, due to the fact that the extracted y1 is always contaminated by other signals. In contrast, the NEWBSE algorithm performs best.

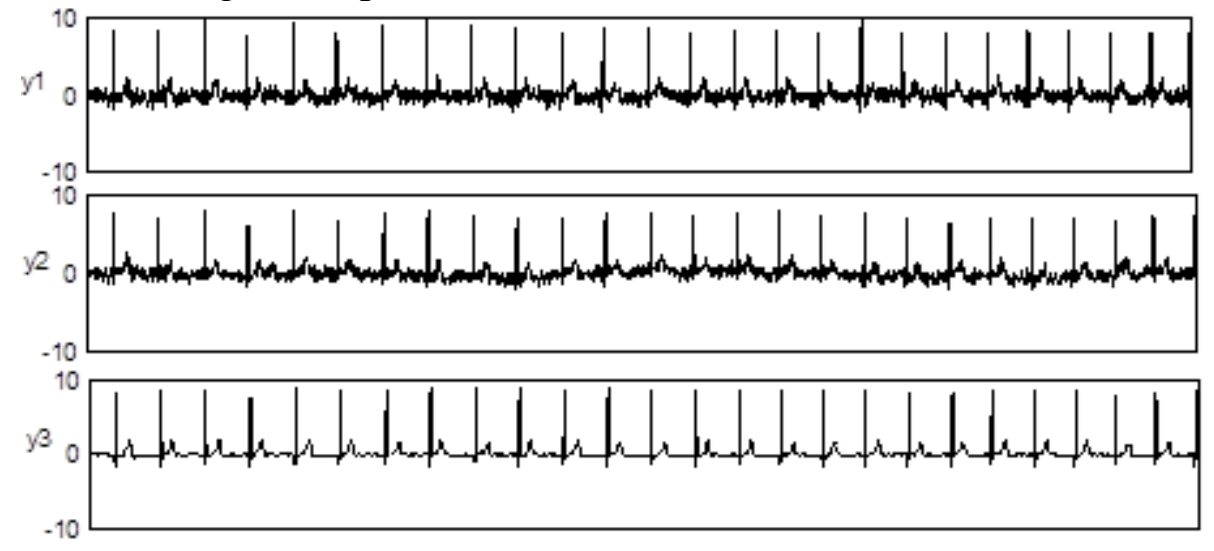

Fig.3 Extraction results on synthetic data

\section{Summary}

A learning algorithm associated with kurtosis has been presented in this paper. It can recover one source signal owning the maximum absolute value of kurtosis among all original sources. In fact, after a deflation process, the extracted signal can be removed from its mixture. The remained signals then can conduct another extraction process to recover the second signal. Thus our method is adaptive and widely applicable.

\section{Acknowledgements}

This work is supported by the National Natural Science Foundation of China (11473019), Natural Science Foundation of Shandong (ZR2014AM015).

\section{References}

[1] C. J. James and C. W. Hesse, Independent component analysis for biomedical signals, Physiological Measurements, vol.26, pp.15-39, 2005.

[2] A. K. Barros and A. Cichocki, Extraction of specific signals with temporal structure, Neural Computation, vol.13, pp.1995-2003, 2001.

[3] Y. J. Zhao, B. Q. Liu and S. Wang, A robust extraction algorithm for biomedical signals from noisy mixtures, Frontiers of Computer Science in China, vol.5, no.4, pp. 387-394, 2011.

[4] Z. L. Zhang and Y. Zhang, Extraction of temporally correlated sources with its application to non-invasive fetal electrocardiogram extraction, Neurocomputing, vol.69, pp. 894-899, 2006.

[5] T. Tsalaile, R. Sameni and S. Sanei, Sequential blind source extraction for quasi-periodic signals with time-varying period, IEEE Transactions on Biomedical Engineering, vol.56, no.3, pp.646-655, 2009.

[6] W. Lu and J. C. Rajapakse, ICA with reference, Neurocomputing, vol.69, pp. 2244-2257, 2006.

[7] Z. L. Zhang, Morphologically constrained ICA for extracting weak temporally correlated signals, Neurocomputing, vol.71, pp. 1669-1679, 2008.

[8] R. Llinares, J. Igual, A. Salazar and A. Camacho, Semi-blind source extraction of atrial activity by combining statistics and spectral features, Digital Signal Processing, vol.21, pp.391-403, 2011.

[9] D. De Moor (Ed.), Daisy: database for identification of systems, 1997, URL: http://www.esat.kuleuven.ac.be/sista/daisy. 